\title{
Dissection of Extended Middle Fossa and Anterior Petrosectomy Approach
}

\author{
Tetsuro Sameshima
}

\subsection{Introduction}

The extradural subtemporal approach through the middle fossa (MF) has become one of the most frequently used operative procedures in contemporary skull base of surgery. This approach is commonly used for exposing lateral wall of the cavernous sinus, to resect anterior petrous bone or to open the internal auditory canal. The MF surgery will be utilized for excision of intracanalicular acoustic neuromas, petrous and infracavernous chordomas, trigeminal neuromas, and small to medium size petroclival meningiomas. Full understanding of microanatomical structure of the cavernous sinus region, middle fossa, and the rhomboid construct is essential for this approach. Through this exercise the surgeon will acquire operative techniques to perform the anterior petrosectomy and also to expose the IAC through the middle fossa.

T. Sameshima

Department of Neurosurgery,

Hamamatsu University School of Medicine,

University Hospital, Hamamatsu city, Japan

\subsection{Standard Middle Fossa Approach}

\subsubsection{Steps of the Surgery}

\subsubsection{Incision, Craniotomy, and Dural Elevation}

The cadaver head is held with lateral position and surgeon at the vertex. The incision begins in the preauricular crease at the zygoma root and continues in a curvilinear fashion past the level of the squamosal suture. The incision extends and cuts the skin and subcutaneous layer to expose temporalis fascia (Fig. 9.1a).

Blunt dissection and a self-retaining retractor expose the temporal muscle. Temporal muscle is split along posterior margin of the skin incision and pulled using skin hooks to expose the temporal squama. A $5 \mathrm{~cm}$ by $5 \mathrm{~cm}$ temporal bone is cut which is placed two-third anterior and one-third posterior to the root of zygoma using a highspeed drill with suction irrigation (Fig. 9.1b). The inferior edge of the craniotomy, especially the inner table of temporal bone, is drilled away to make this opening even with the middle cranial fossa floor level along the zygoma root. Subtemporal inner plate at the middle fossa base must be shaved entirely flat (Fig. 9.1c, d).

The temporal lobe dura is dissected along the middle fossa floor starting from posterior to anterior. Foramen spinosum and foramen ovale are then skeletonized, and middle meningeal artery 
(MMA) is coagulated and divided. The dura propria is elevated from trigeminal 3rd branch (V3) at the foramen ovale using No.15 blade scalpel and a sharp dissector. Identify the location of the geniculate ganglion and greater superficial petrosal nerve (GSPN). This dural elevation is important as $15 \%$ of geniculate ganglion lie under a dehiscence in the middle fossa floor. In surgery, the position of geniculate ganglion can be made sure by stimulation with a facial nerve stimulator. The surgeon needs to assume the approximate position of the cochlea and how far it is from the geniculate ganglion and the petrous carotid artery (C6). The petrous ridge is identified medially, and the Fukushima middle fossa rigid tapered retractor is positioned (Fig. 9.1e).

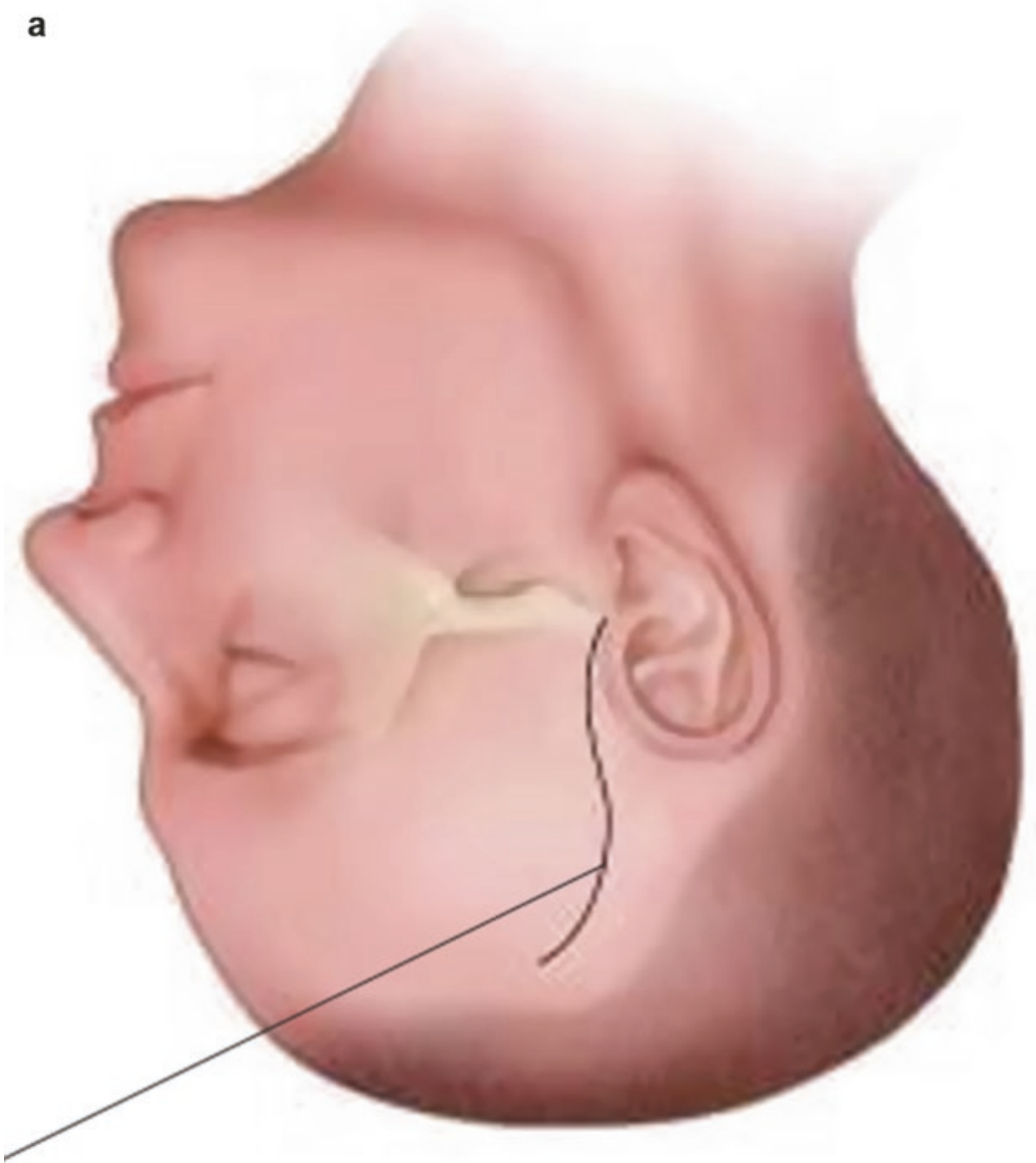

Fig. $9.1(\mathbf{a}-\mathbf{e})$ 
Fig. 9.1 (continued)
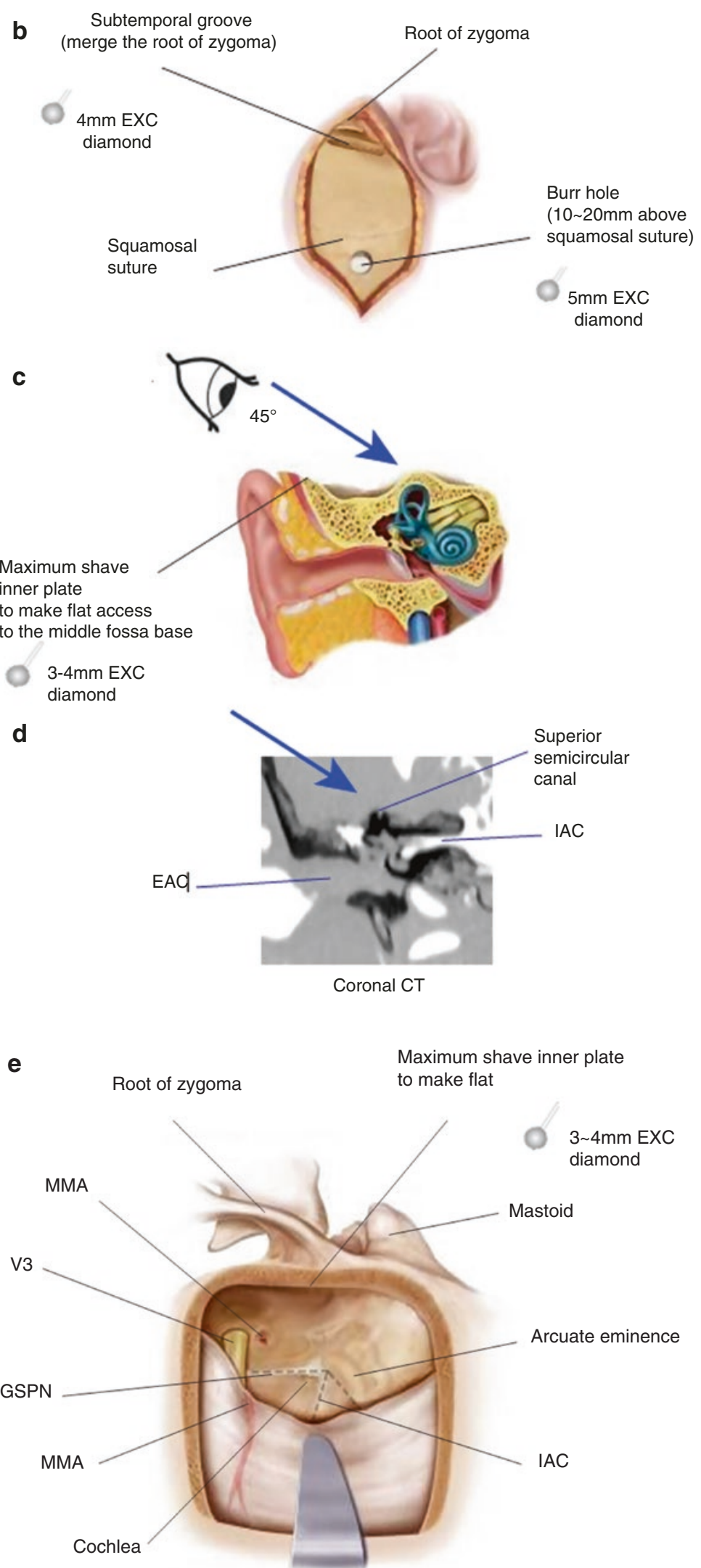
The arcuate eminence covering the superior semicircular canal is identified. The bone of the arcuate eminence is gently drilled off until the "blue line" of the superior semicircular canal is seen. Violating the canal will usually lead to deafness. In the fixed specimen, the line is not necessarily blue but green. Try to thin the denser cortical bone that surrounds the membranous labyrinth using a smooth diamond burr. Be careful that arcuate eminence is not always precisely overlying the canal. MF hemostasis must be complete and quick with diamond drill heat, touch spring coagulation, bone wax, bipolar, and surgicel.

\subsubsection{IAC Drilling}

Surface landmarks can be misleading. The surgeon must be oriented to the approximate location of IAC before drilling commences. The orientation of the IAC is a direct medial extension of the external ear canal (EAC). Identification of the GSPN provides a guide to the location of the geniculate ganglion and arcuate eminence, which can be used to approximate the location of the IAC.

Drilling starts anteriorly to and medially near the porus trigeminus along the petrous ridge. To facilitate a wider angle to the middle fossa with the microscope, we recommend using a pair of $2 \mathrm{~mm}$ and $4 \mathrm{~mm}$ tapered tip rigid steel retractor blades with the retractor tip wedged at the base between the petrous ridge and the dura. In that way we can retract effectively the temporal basal dura to obtain maximum surgical exposure toward the middle fossa. Gradual gentle drilling is recommended with constant cooling irrigation using a power diamond drill bit laterally toward the petrous carotid and posteriorly toward the arcuate eminence to expose the IAC (Fig. 9.2a-d).

There are four surgical techniques to identify the IAC during middle fossa approach. The classic technique described by William House follows the GSPN to geniculate ganglion and then approaches to labyrinthine segment of the facial nerve into the fundus of the internal auditory canal. Then continue to skeletonize the internal auditory canal (Fig. 9.2e). The technique popularized by Ugo Fisch is termed the meatal plane approach (Fig. 9.2f), in comparison to other approaches (Fig. 9.2e-h). In this technique, first identify superior semicircular canal which looked like a "blue line" (leaving a thin layer of bone on the top membranous labyrinth). At this step, a $60^{\circ}$ viewpoint from the amputated part of the superior

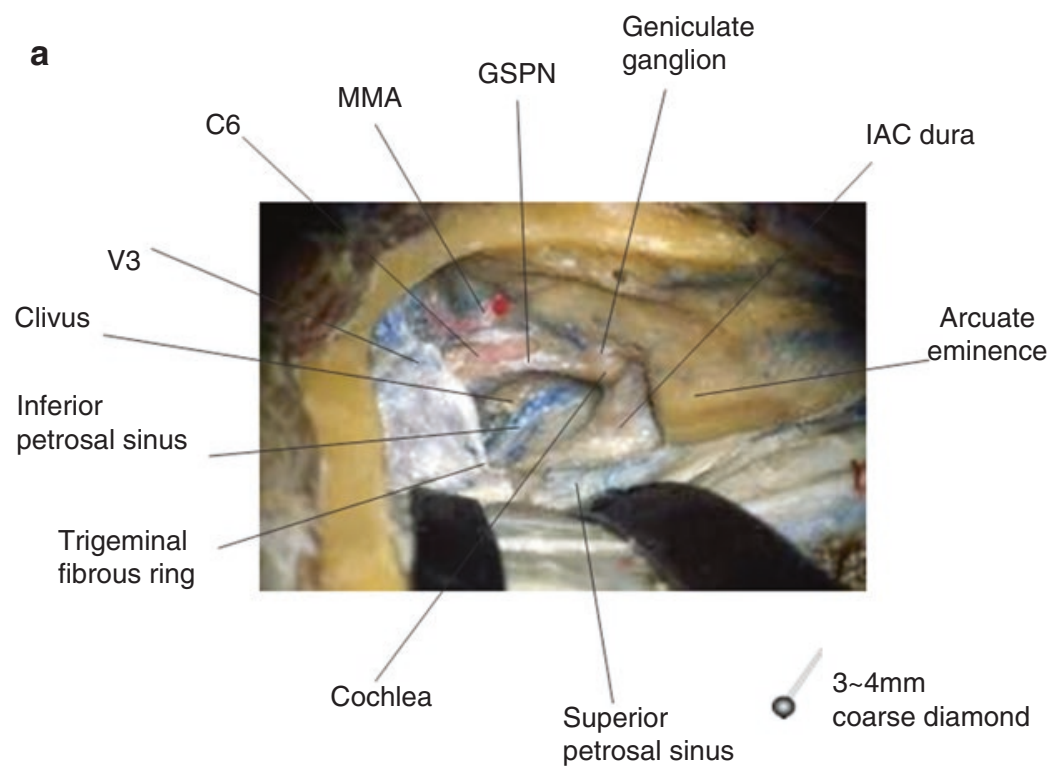

Fig. $9.2(\mathbf{a}-\mathbf{h})$ 


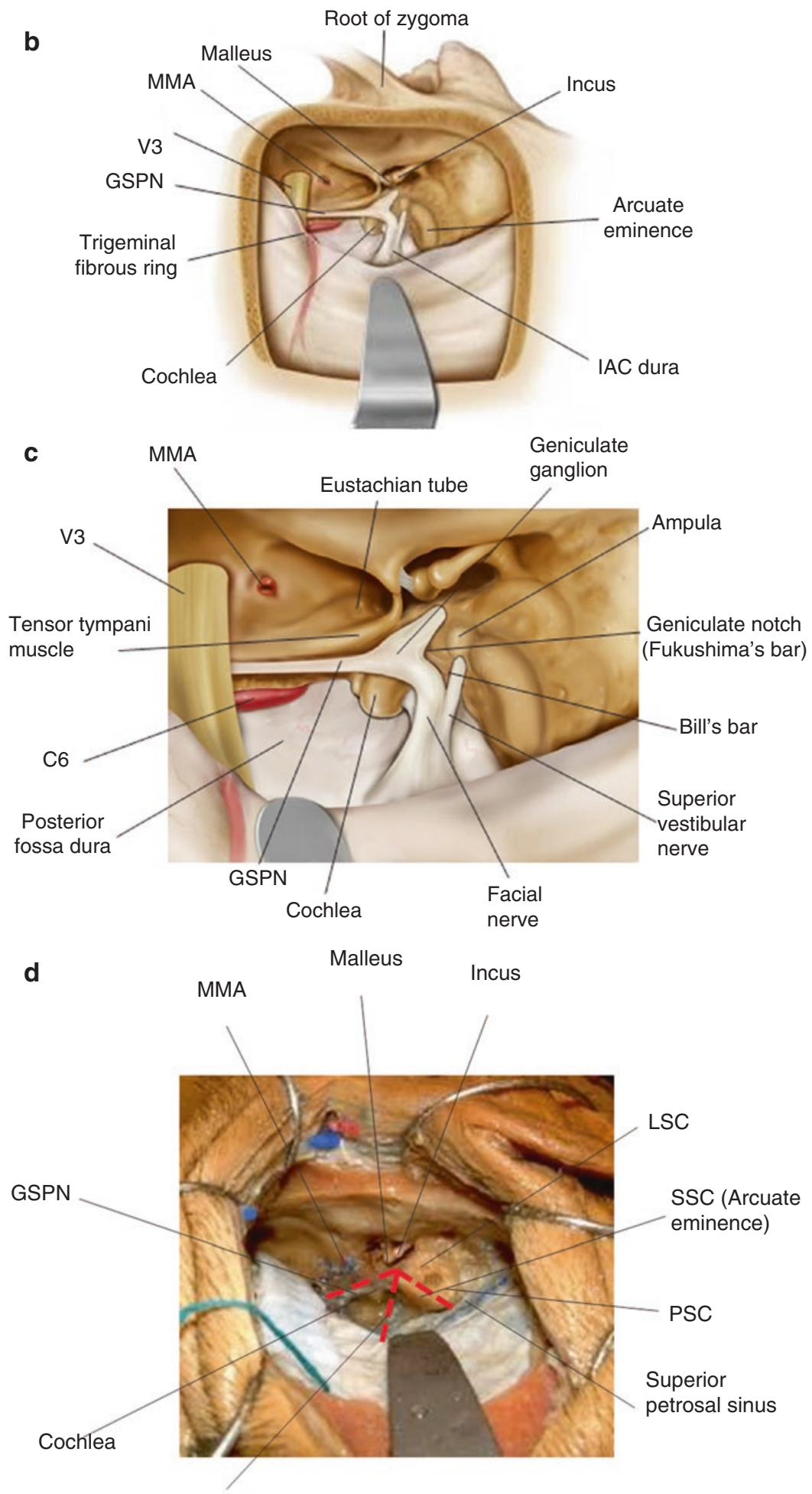

IAC dura

Fig. 9.2 (continued) 
Fig. 9.2 (continued)

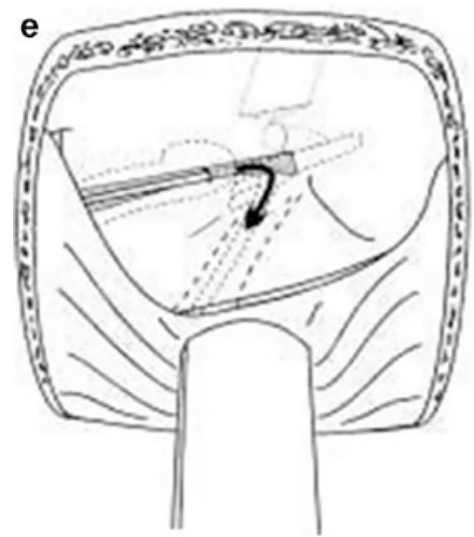

William House

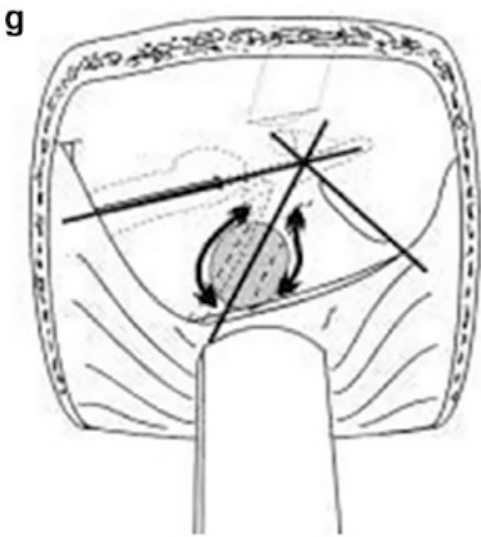

Garcia-Ibanez

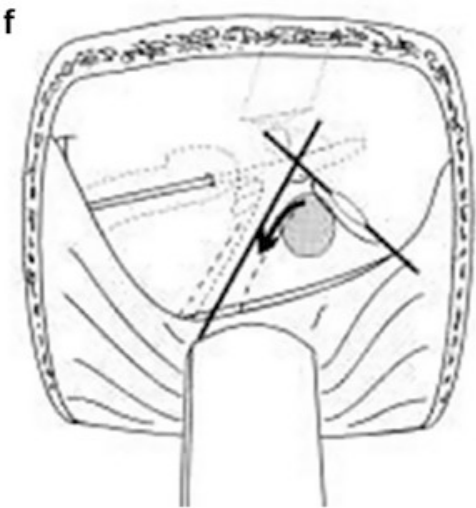

Ugo Fisch

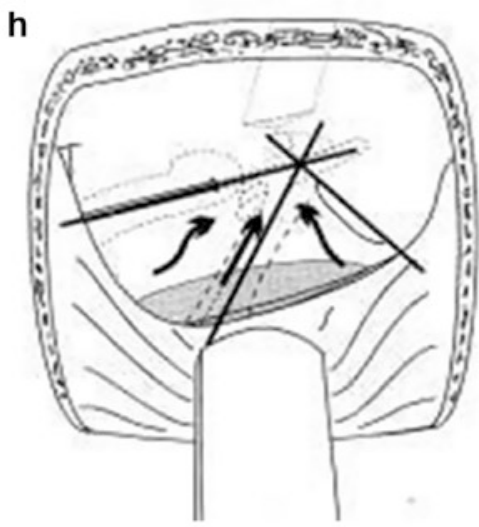

Mario Sanna canal outlines a safe zone to drill the IAC. The third approach popularized by Garcia Ibanez is a medial drilling technique (Fig. 9.2g). After identifying the geniculate ganglion and arcuate eminence, the surgeon begins drilling medially on a line bisecting the angle between the arcuate eminence and the GSPN. It is safest to find the IAC by drilling close to the petrous ridge (Fig. 9.2h). Near the porus, the IAC dura is safe to be skeletonized around $270^{\circ}$. As the drill proceeds toward the fundus of the IAC, only the roof of the canal can be removed. Overly aggressive drilling of IAC fundus will disrupt cochlea anteriorly or the superior semicircular canal posteriorly. The latter technique is advantageous because the vulnerable inner ear part (cochlea and labyrinth) are away from the initial drilling prior to the IAC identification. While keeping in mind the location of the cochlea, the final drilling at the isthmus between the cochlea and arcuate eminence reaching toward Bill's bar is continued.

\subsubsection{IAC Dura Incision and Tumor Removal}

The dura should be maintained intact until the IAC exposure is complete. Drilling after opening the IAC dura risks damage to the IAC contents. After full skeletonization of the IAC (Fig. 9.3b), the dura is cut with reversed $\mathrm{T}$ shape along petrous ridge and then along the IAC. With the dural incision, be careful not to injure branches from the AICA vessel (Fig. 9.3a).

In acoustic tumor surgery, the dura is reflected anteriorly and posteriorly to expose the intracana- 
licular acoustic tumor (Fig. 9.3c). After locating the thin facial nerve both anatomically by the high magnification microscope and physiologically with $0.05 \mathrm{~mA}$ facial nerve monitor, we then start to dissect the facial nerve from the tumor capsule using a sharp knife, sickle knife, and angle-type hooks. The surgeon always identifies the meatal loop and eventually the labyrinthine artery. Almost always the most important key is the identification of the remaining vestibular nerve which forms a pseudocapsule tumor; and once the surgeon is able to identify this separation plane, the
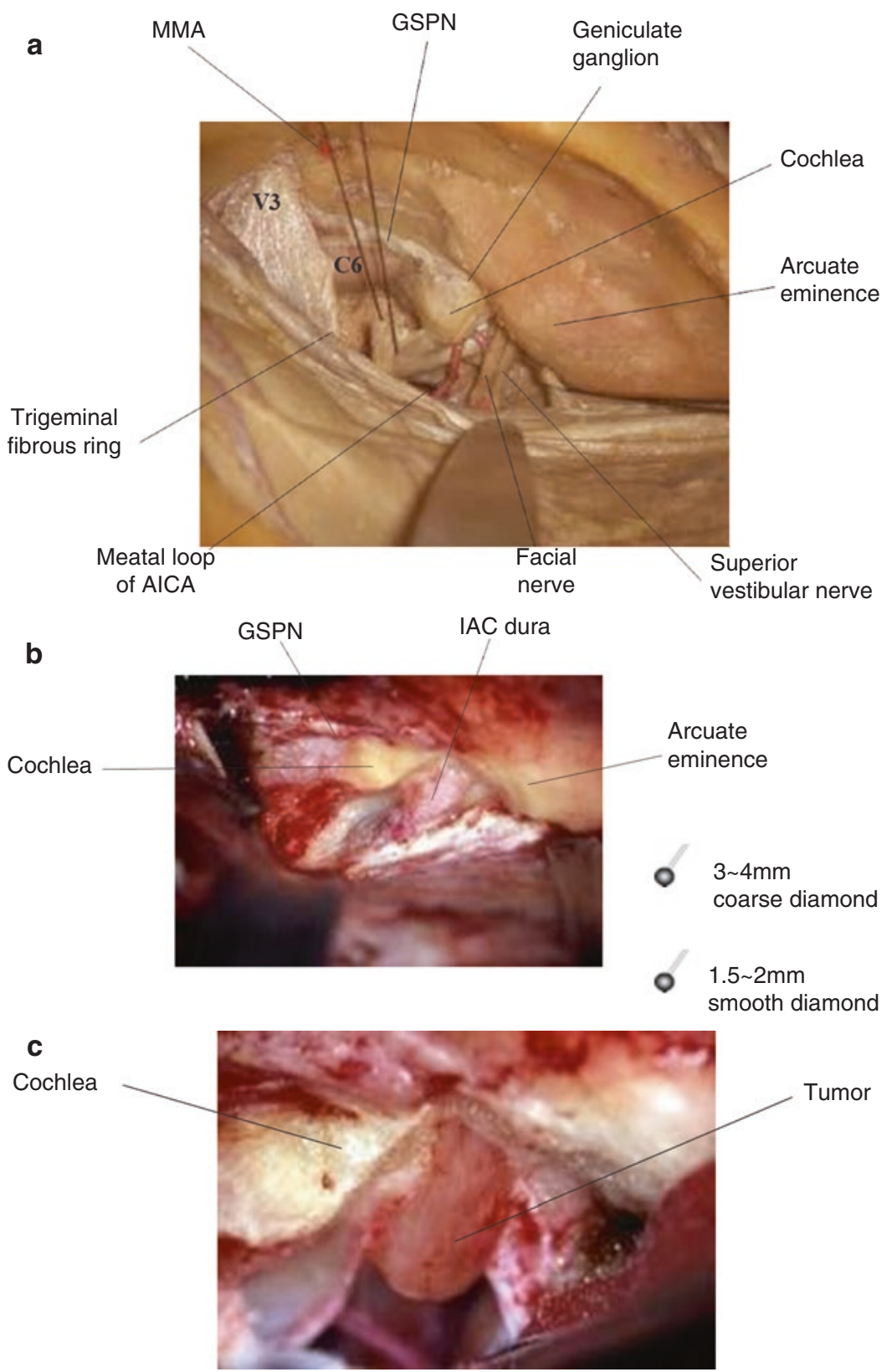

Fig. $9.3(\mathbf{a}-\mathbf{d})$ 
Fig. 9.3 (continued)

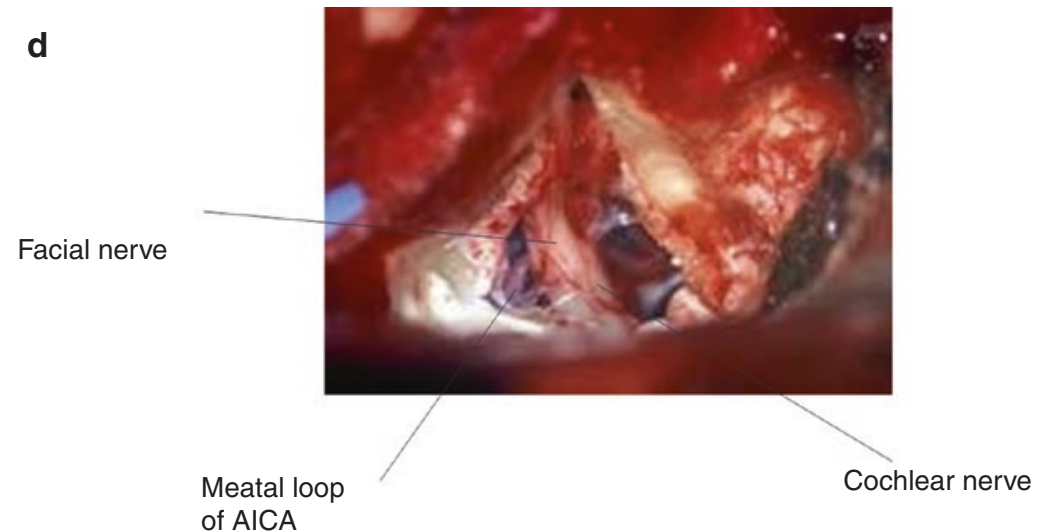

tumor capsule will be easily elevated using various supermicro $\mathrm{CP}$ angle instruments. The tumor's center has to be piecemeal removed, and then tumor portion at IAC can be elevated safely off from the thin facial nerve and then farther ventral and in the deeper part of surgical site from cochlear nerve. The most important technical tip is to maintain a bloodless, clean operative field with high magnification to keep the microanatomy (Fig. 9.3d).

\subsection{Extended Middle Fossa and Anterior Petrosectomy Approach}

Basically, start with middle fossa approach, and then extend the exposure to its anterior part. Petrous ridge is followed more anteriorly exposing porous trigeminus. Dura propria is detached from mandibular branch of trigeminal nerve. The drilling needs to be done at the rhomboid area (between IAC and ICA), exposing the ascending segment of the internal carotid and the whole path of abducens nerve including Dorello's canal. Here are some example cases that could be done with this approach (Fig. 9.4a-c).

\subsubsection{Steps of the Surgery}

\subsubsection{Incision, Scalp Reflection, and Craniotomy}

Place the cadaver head in the lateral position, and position yourself at the vertex. Make the skin inci- sion in the shape of a question mark concave anteriorly, as illustrated (Fig. 9.4d). Incise the fascia overlying the root of the zygomatic process, and elevate it, using a periosteal dissector, from the lateral and medial surfaces of this structure. The goal is to free the temporal muscle from the zygomatic root, allowing the surgeon to mobilize the muscle anteriorly. This maneuver will help to provide a better view all the way at the floor of middle fossa, without needing to perform a zygomatic osteotomy.

Make a burr hole at superior to the squamosal suture, and drill a groove in the bone above the root of the zygoma using $4 \mathrm{~mm}$ extracoarse (EXC) diamond drill. Using a craniotome cut an approximately $5 \mathrm{~cm} \times 5 \mathrm{~cm}$ four-sided bone flap; the position of the bone flap is about two-thirds in front of the external auditory canal (Fig. 9.4e). Using a small periosteal dissector, separate the dura from the bone along inferior edge of the craniotomy until the middle fossa floor is exposed. Using a cutting burr or a rongeur, remove any remaining bone to the level of the middle fossa floor. This will provide an unobstructed, flat view across the floor.

\subsubsection{Dural Elevation and Identification of "Rhomboid" Structure}

Retract the dura along the petrous ridge. The petrous ridge is composed of two ridges (medial and lateral). Identify the arcuate eminence as a primary landmark. After exposing the arcuate eminence, elevate the dura moving anteromedially to expose the greater superficial petrosal nerve (GSPN) and also tegmen tympani. The 

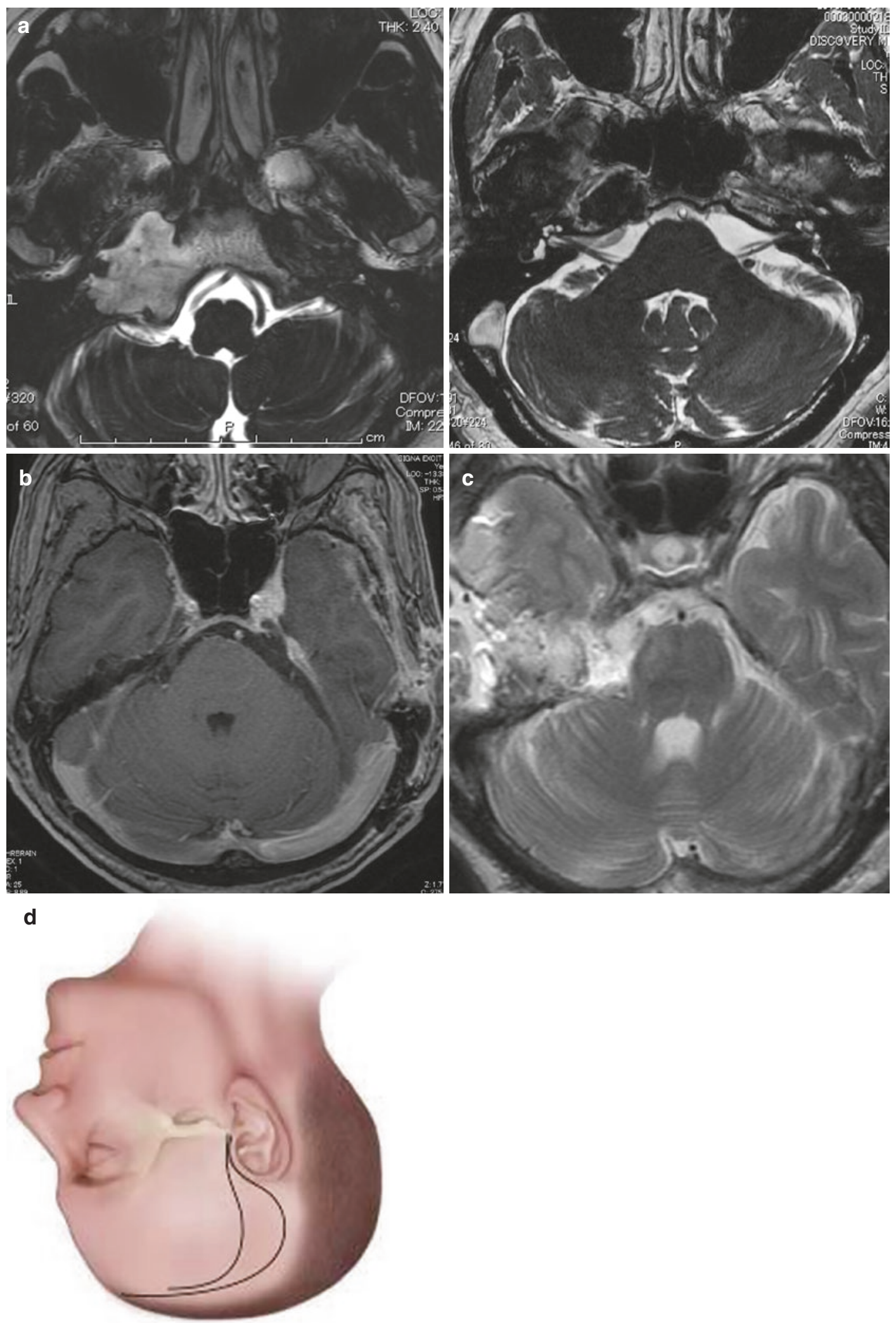

Fig. $9.4(\mathbf{a}-\mathbf{e})$ 


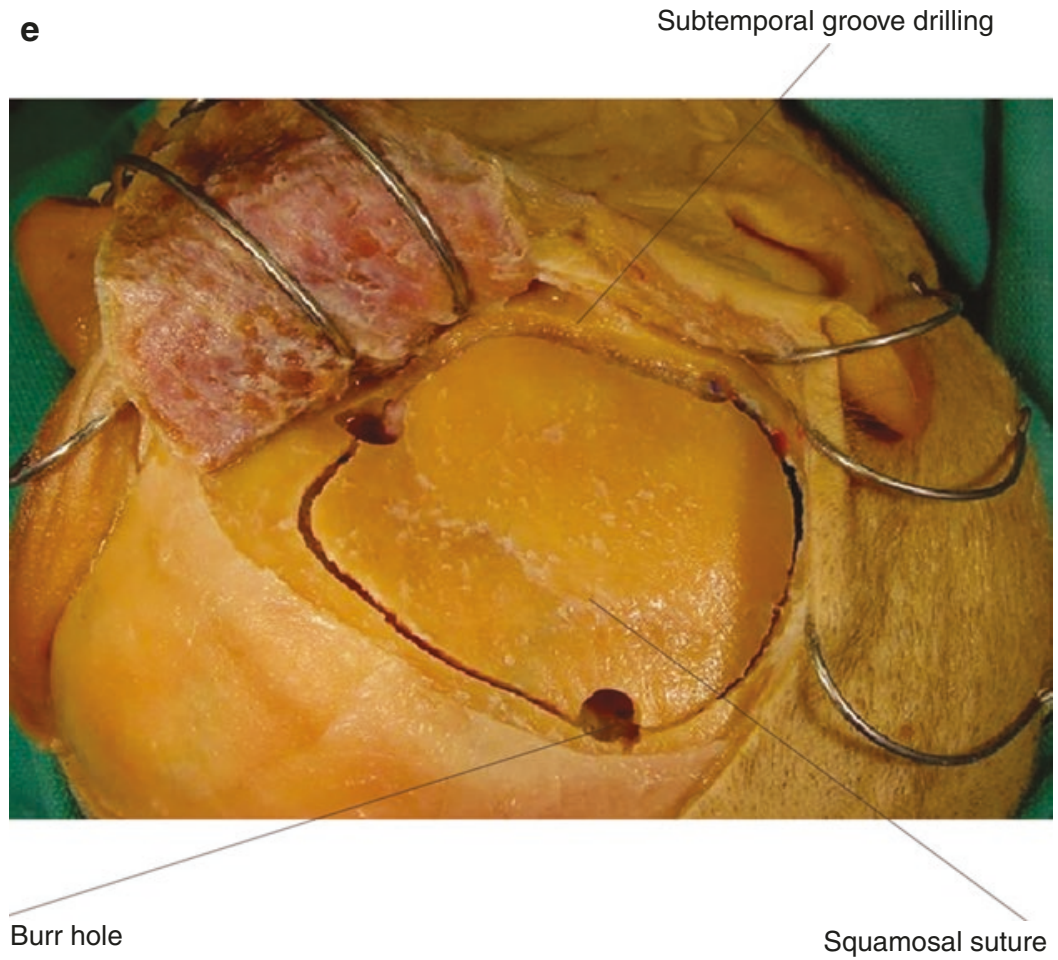

Fig. 9.4 (continued)

GSPN lies in the major petrosal groove and is shielded by thin layer of connective tissue, and it is continuous with the periosteum, which makes identification of the nerve dependent upon removal of this covering. Detach the dura to identify the middle meningeal artery that exits from foramen spinosum. Divide the artery at its entrance into the cranial vault. Now identify and expose third division of trigeminal nerve when it goes in to the foramen ovale.

Elevate the dura in posterior direction toward petrous ridge to expose bone between arcuate eminence and the trigeminal impression. Place two self-retaining tapered retractors to hold the temporal dura away from the middle fossa floor.

Sharply free the dura from the lateral trigeminal complex by developing the plane between the temporal dura and the connective tissue sheath of the nerve. This maneuver will increase the width of the extradural corridor through which the procedure is performed.

The middle fossa anatomical landmark that defines "rhomboid" complex now can be recog- nized (Fig. 9.5a, b). Few important parts are the intersections between GSPN and trigeminal nerve, between petrous ridge and arcuate eminence (AE), and between projected line along the axes of the GSPN and AE, and it is also very important to recognize the porus trigeminal.

This complex, projected obliquely toward the clivus through the petrous pyramid, delimits the volume of bone which will be removed.

\subsubsection{Extradural Bone Removal}

The goal of this stage of the operation is to create a maximal window in the anteromedial petrous pyramid while preserving the neural and vascular structures of the temporal bone. This objective is best accomplished by proceeding with the extradural bone removal in a precise, stepwise fashion.

Begin by drilling gently the medial two-thirds of IAC. Bisect the projected line along the axes of GSPN and AE. Start drilling by using 3 or $4 \mathrm{~mm}$ coarse diamond burr along midpoint of the bisection axis. We will find the dura that covers IAC 
Fig. $9.5(a, b)$

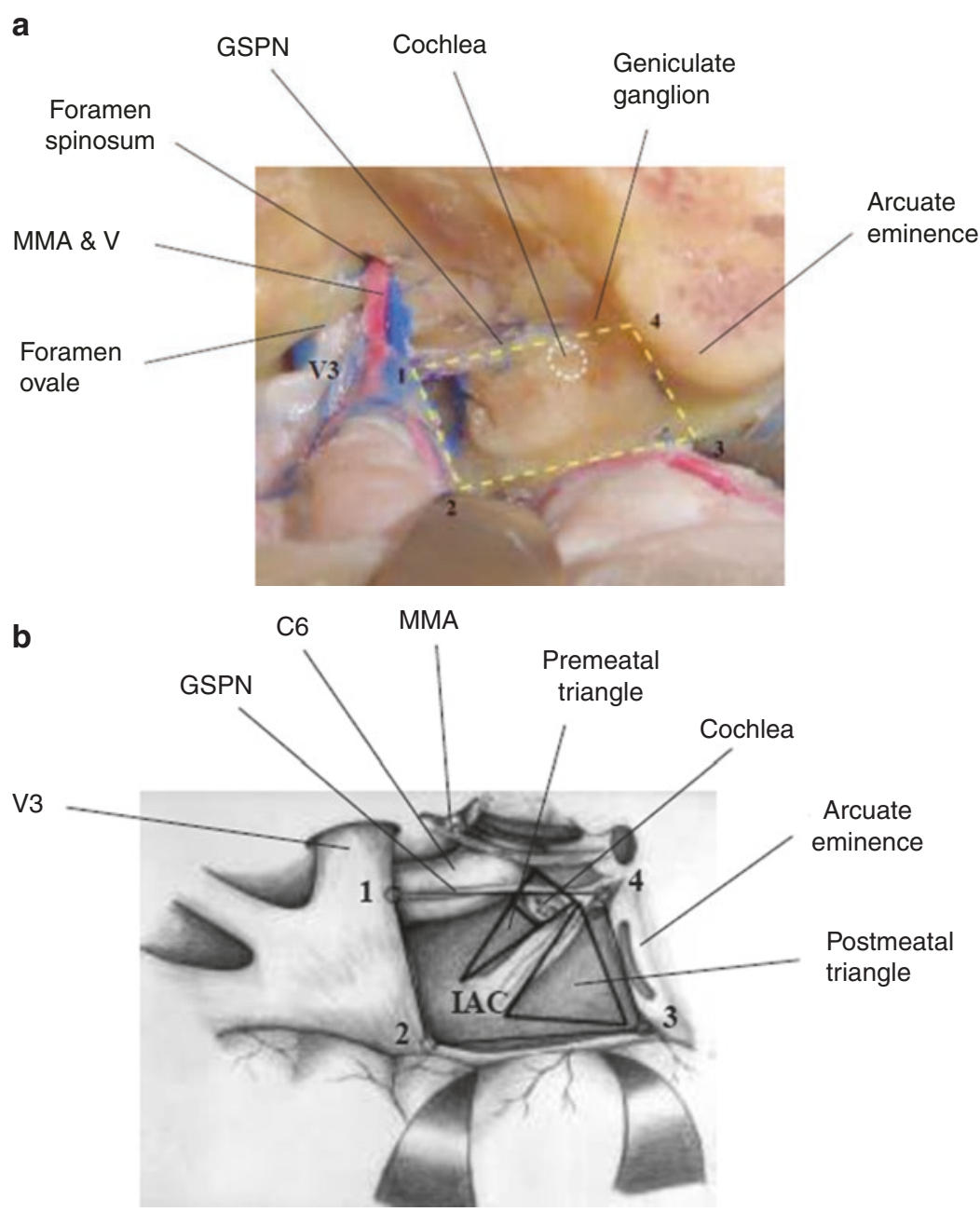

after we removed about 3-4 $\mathrm{mm}$ of the bone. Keep exposing and following dura that covers the medial IAC toward petrous ridge. The dura will flare at the porus acusticus, which will signal medial extent of the canal. Remove several millimeters of bone on the anterior side of the IAC, in the direction of the trigeminal complex.

Next, the GSPN and geniculate ganglion are addressed. The GSPN run through bony canal and come out at facial hiatus to continue its medial course in the major petrosal groove. With the medium diamond burr, the GSPN is gently exposed posteriorly to the facial hiatus, moving toward the geniculate ganglion. Expose the geniculate ganglion using light strokes with the smooth diamond burr, preserving a thin shell of bone over the ganglion. Uncover the entire IAC toward the geniculate ganglion. As you approach the fundus of the IAC, the opening should be no more than the width of the canal. Remember that the facial nerve sweeps upward to join the geniculate ganglion as it passes the cochlea. This moves the intracanalicular facial nerve more superficial as the fundus of the IAC is exposed.

Two of the three key landmarks, which locate the cochlea, are now identified: the geniculate ganglion and the porus acusticus. To identify the third important landmark used to avoid opening the cochlea (the intrapetrous carotid artery genu), uncover the internal carotid artery in the posterolateral triangle close to V3. Still using the medium diamond burr, expose the artery by removing the bone between the GSPN and the foramen spinosum. Divide the GSPN near its intersection with 
the trigeminal complex and reflect it posteriorly. Expose the carotid artery from where it crosses underneath the fifth nerve to lateral part where tensor tympani muscle crosses over the artery. At the point where the tensor tympani muscle and adjacent eustachian tube cross the artery lies the genu of the internal carotid artery. This completes the identify cation of the three defining landmarks of the premeatal triangle, where the cochlear resides (Fig. 9.6a-d).

Remove the soft, porous bone between the carotid artery and IAC, avoiding the posterior lateral volume of bone housing the cochlea. Surrounding bone of the cochlea is identified by its
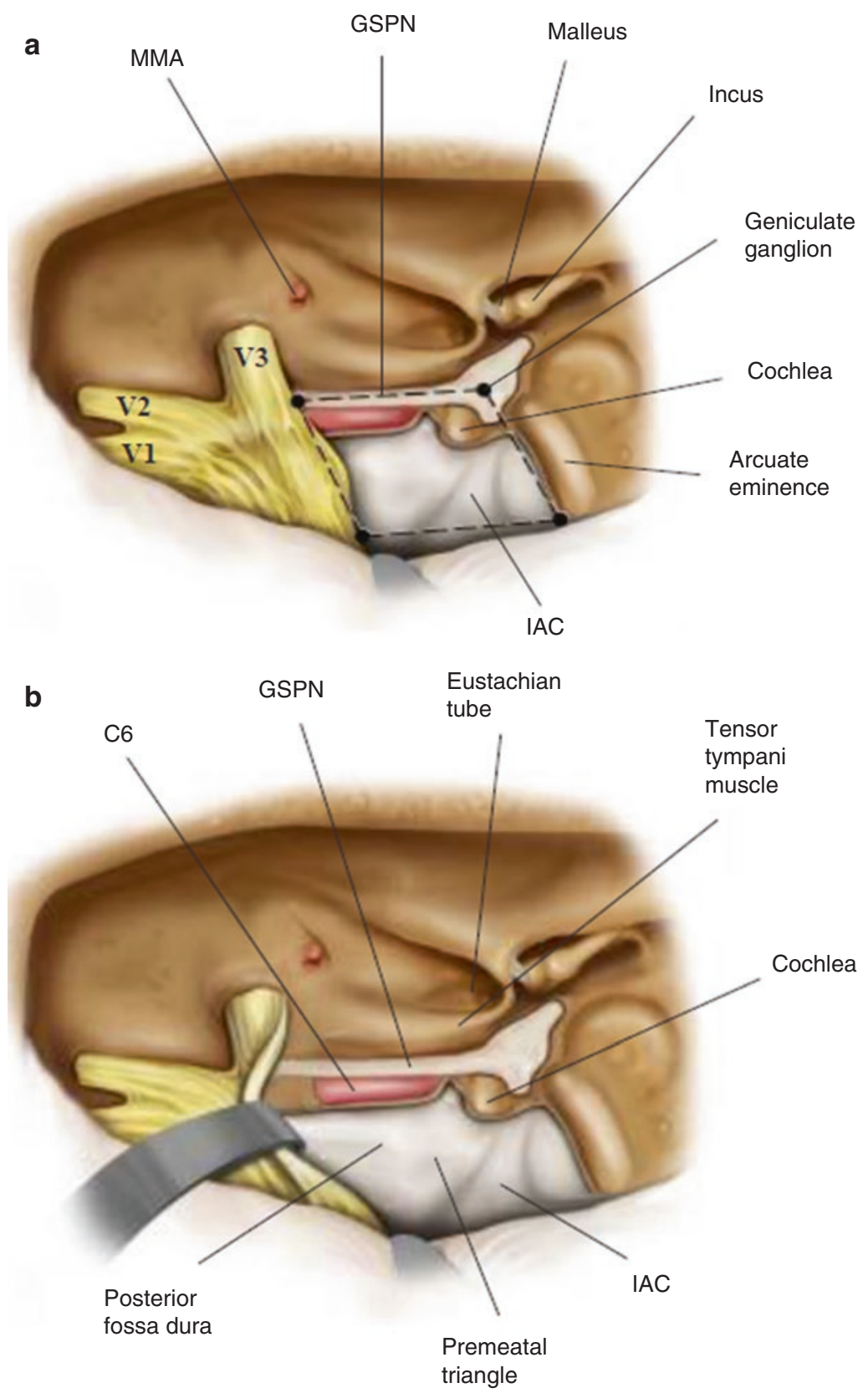

Fig. $9.6(\mathbf{a}-\mathbf{j})$ 


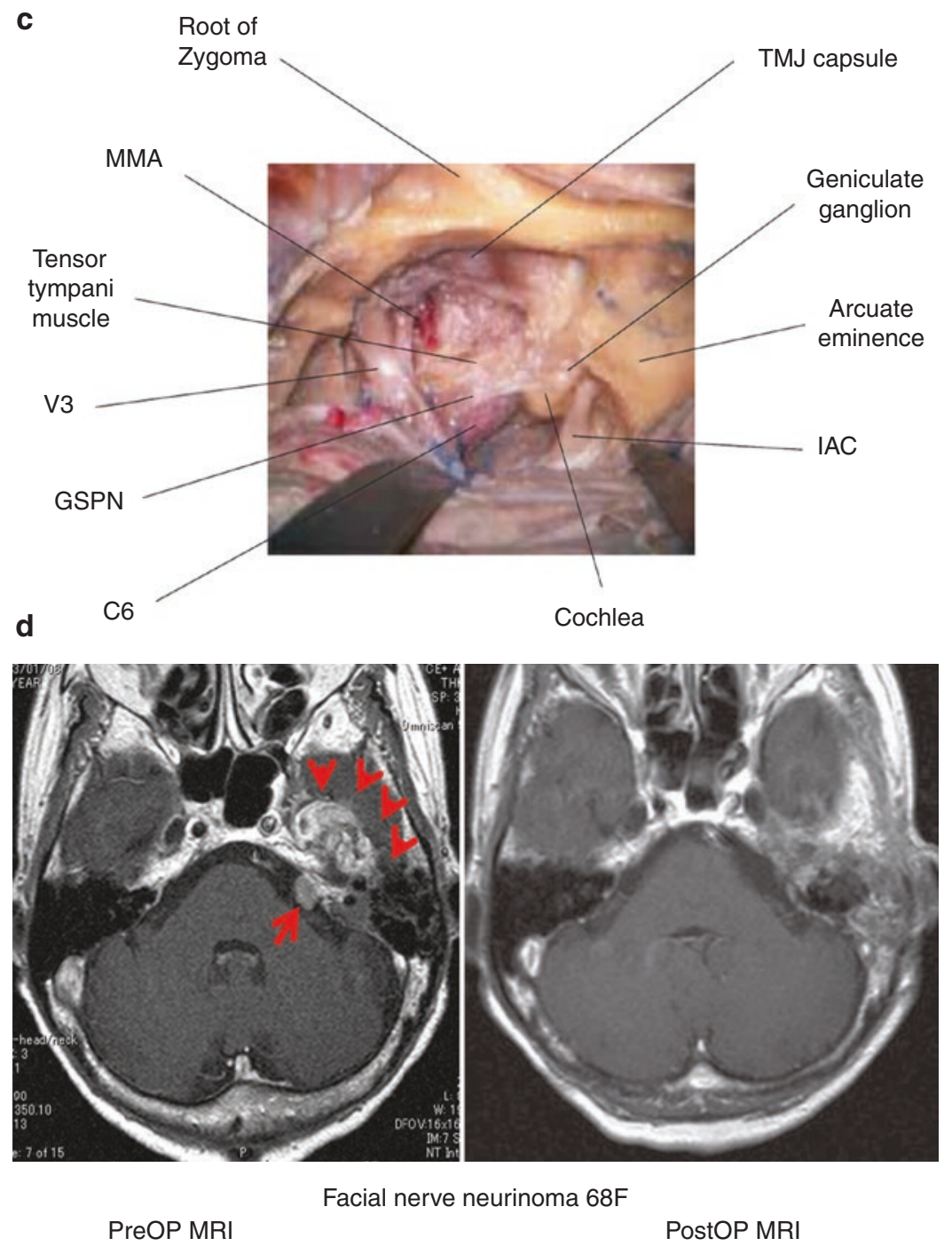

Fig. 9.6 (continued) 

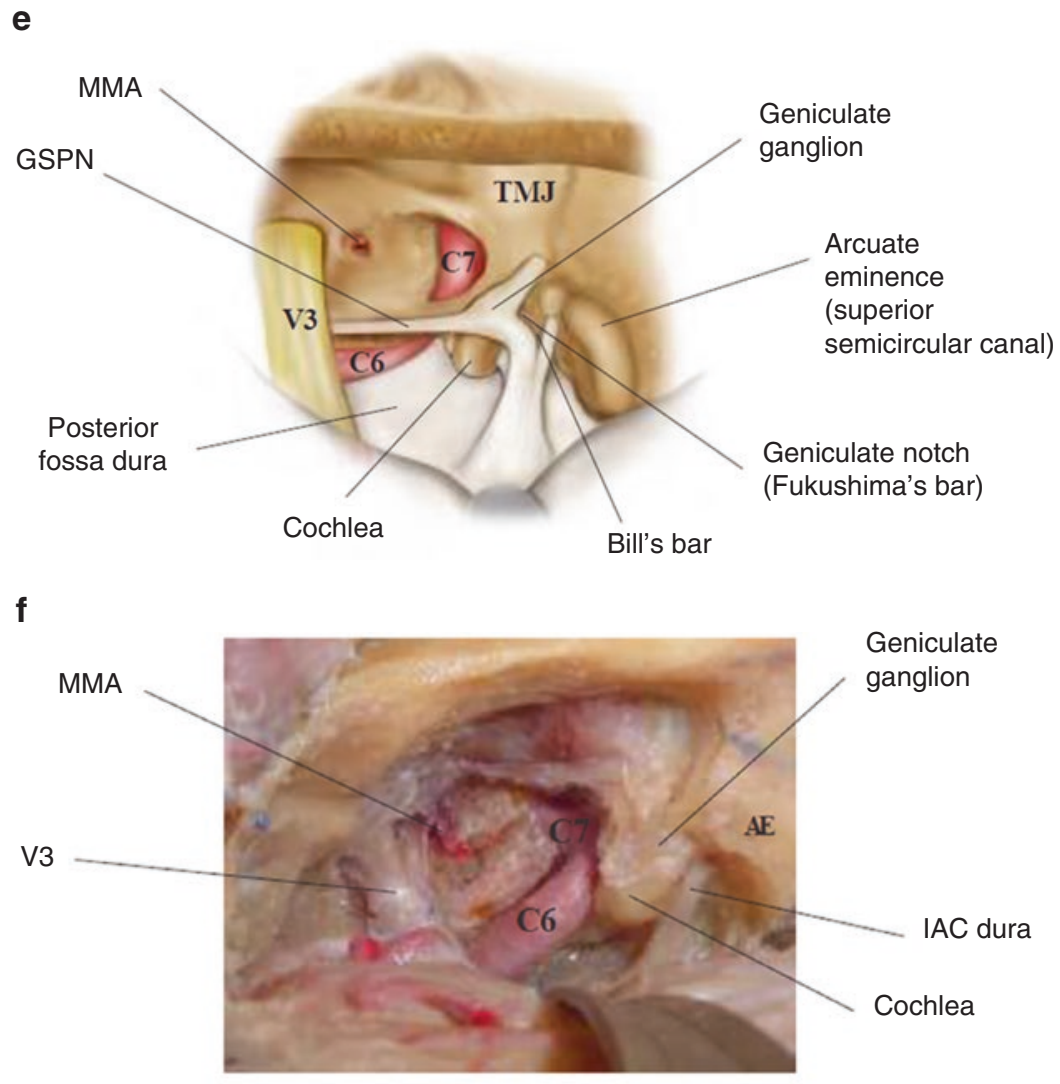

Internal carotid artery (C6-C7) exposure

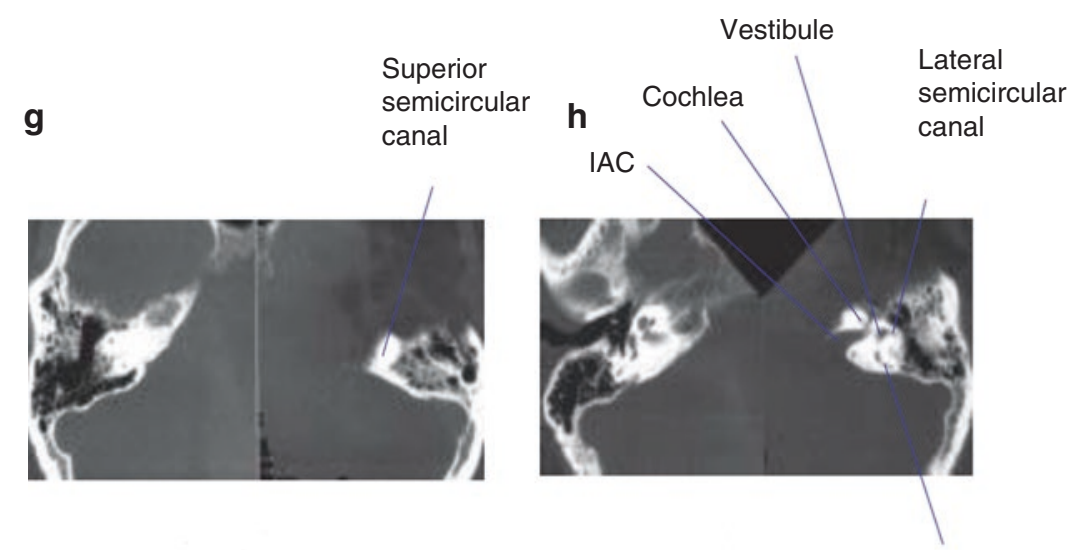

Posterior semicircular canal

Fig. 9.6 (continued) 


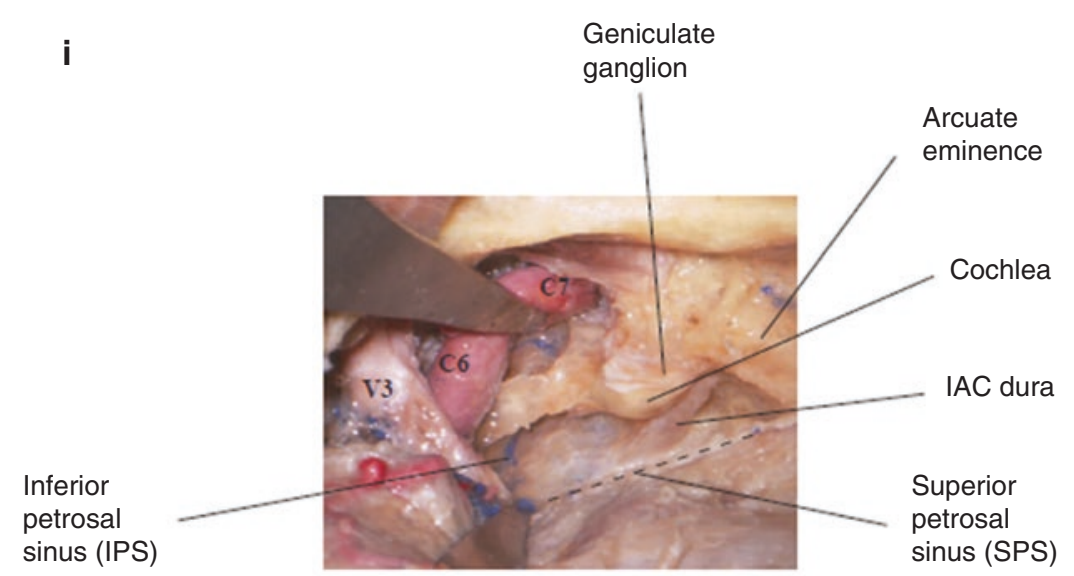

Internal carotid artery translocation \& clivus exposure

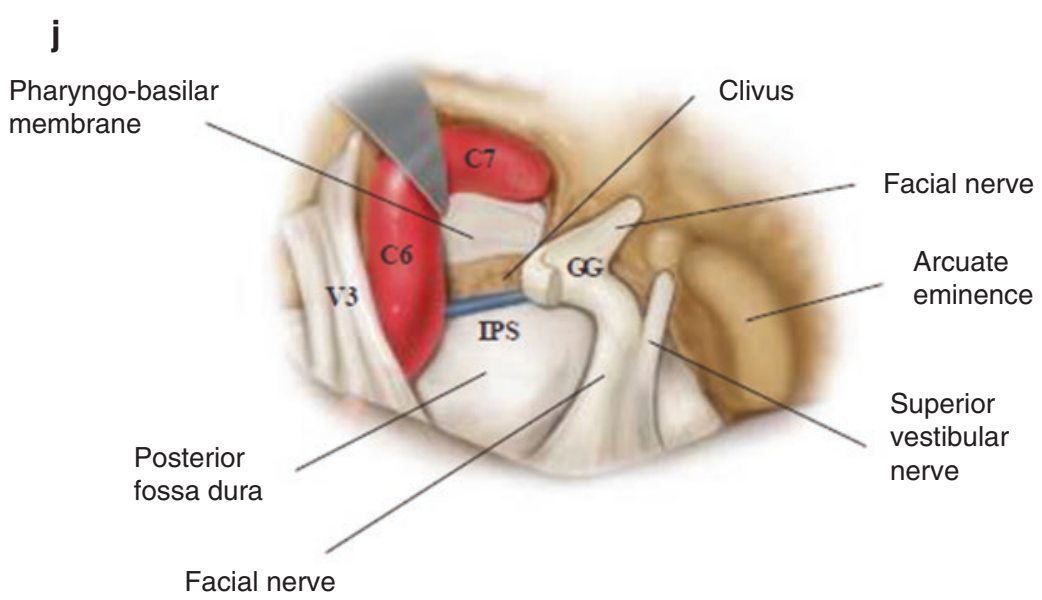

Fig. 9.6 (continued)

compact, nonporous nature. Dura of the posterior fossa is exposed medial and anterior to IAC by drilling away the wedge-shaped bone between superior semicircular canal and IAC (called "postmeatal triangle") using a small or medium diamond burr. Marking the superior semicircular canal with "blue line" color is very helpful, which usually lies under the arcuate eminence, in order to precisely define the lateral border of the triangle. Removal of this volume of bone will effectively unroof approximately $270^{\circ}$ of the circumference of the IAC at the meatus (Fig. 9.6e, f).
Now the final stage of bone removal is performed: exposing posterior fossa dura inferior to IAC by removing the bone between the IAC and the intrapetrous carotid artery. First work medially and then laterally. At the lateral margin, the volume of bone containing the cochlea should be "undercut" in order to maximize exposure of the posterior fossa dura. The cochlea, lying within the basal half of the "premeatal triangle," is quite vulnerable to injury at this stage. Again, the change in the quality of the bone surrounding the cochlea must be rec- 
ognized. Also note that the facial nerve lies in the anterior aspect of the IAC, so be especially careful in removing bone from the posterior premeatal triangle. Expose the dura moving inferiorly to expose the inferior petrosal sinus (IPS). Remove bone across this sinus until the cancellous bone of the clivus is reached.

At the anterior end, remove the bone of the petrous apex by coring out the apical bone next to the foramen lacerum. Staying within the cortical bone, thin the cortex until only a depressible shell remains. Dissect this shell away from the dura using a small sharp dissector. When the apex has been removed, the foramen lacerum will be opened posteriorly. Dissect the residual tip of bone free with a microdissector, and remove it with microrongeurs (Fig. 9.6g-j).

\subsubsection{Dural Opening}

Before opening the dura, we must interrupt superior petrosal sinus (SPS) at the porus trigeminus. Incise the dura superior to SPS starting from porus trigeminus to arcuate eminence. Make a second incision, parallel to the first, inferior to SPS in the posterior fossa dura. Ligate the sinus anteriorly at the porus trigeminus. Make a sagittal incision in the medial tentorium, $8-10 \mathrm{~mm}$ in length. Place a stitch in the lateral corner of the incised tentorium and retract this superiorly.
Open the dura surrounding the trigeminal root at the porus trigeminus. Now incise the posterior fossa dura, at lateral and medial margins of the exposure, toward inferior petrosal sinus (IPS). Incise the dura along the margin of the IPS to completely excise this section of posterior fossa dura. It is important to identify the basilar artery in the deepness of the exposure. The AICA origin should next be located and its course followed to the region of the porus acusticus. The sixth cranial nerve will be seen crossing over the AICA as it ascends toward Dorello's canal (tube). Dorello's canal is located inside the IPS far anteriorly. Note the differences in exposure by rotating the microscope through various angles from medial to lateral (Fig. 9.7a-e).

The extended middle fossa approach allows the surgeon to access the posterior fossa lesions that involve the CPA and extend anteriorly into the cavernous sinus and inferiorly along the prepontine region of the clivus. Simultaneous access is possible for transtentorial tumor extensions and hearing preservation. The major limitation of this technique is the amount of temporal lobe retraction necessary which can be much more extensive than that required for a standard middle fossa approach. Several examples of cases include trigeminal neurinoma, Meckel's cave meningioma, and basilar trunk aneurysm (Fig. 9.7f-h).

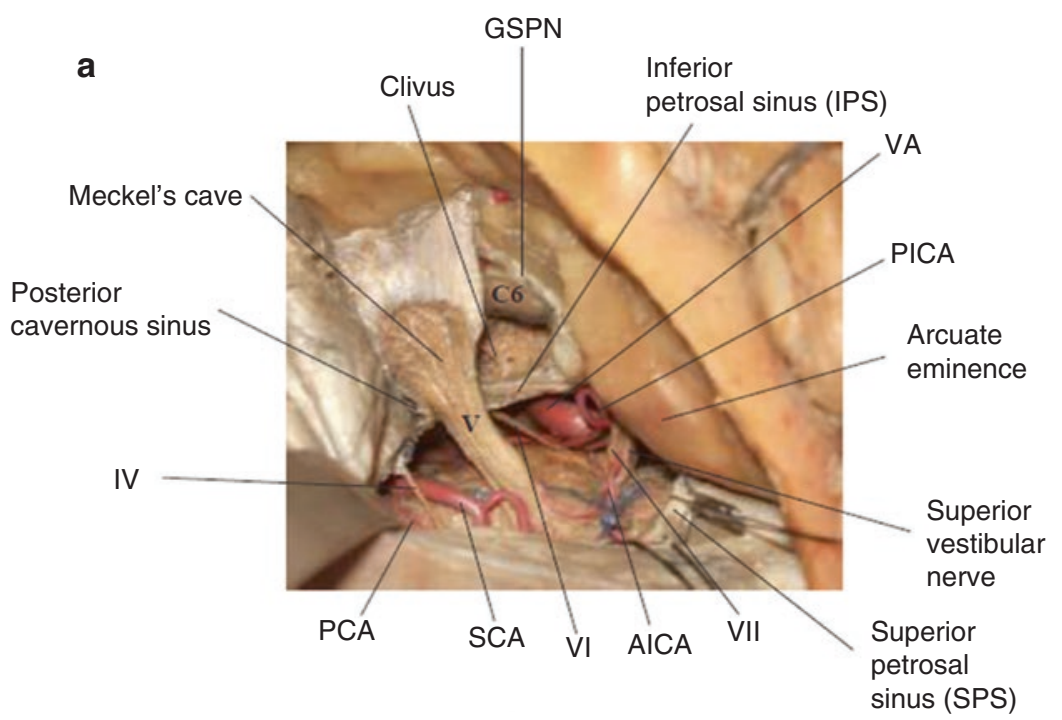

Fig. $9.7(\mathbf{a}-\mathbf{h})$ 

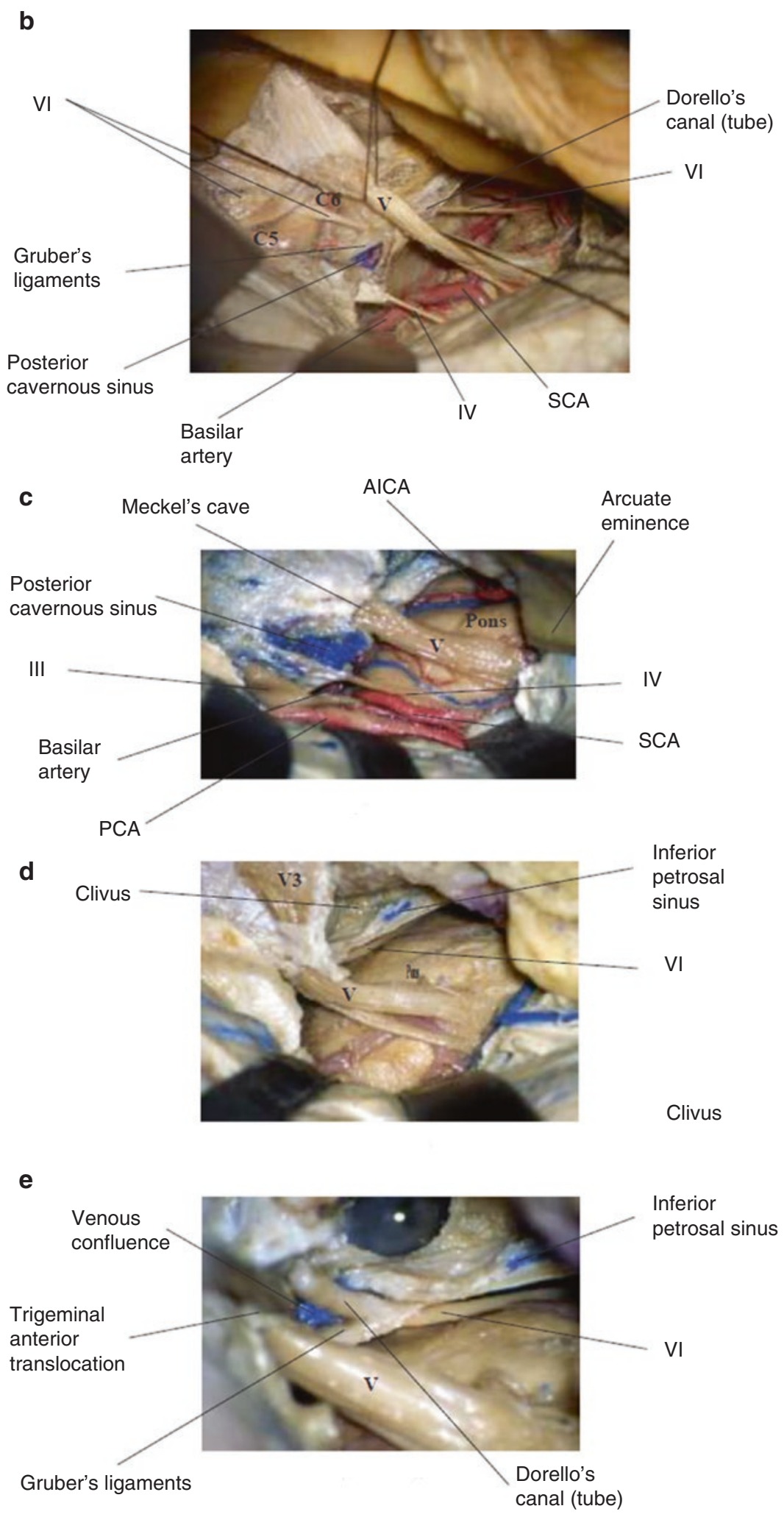

Fig. 9.7 (continued) 


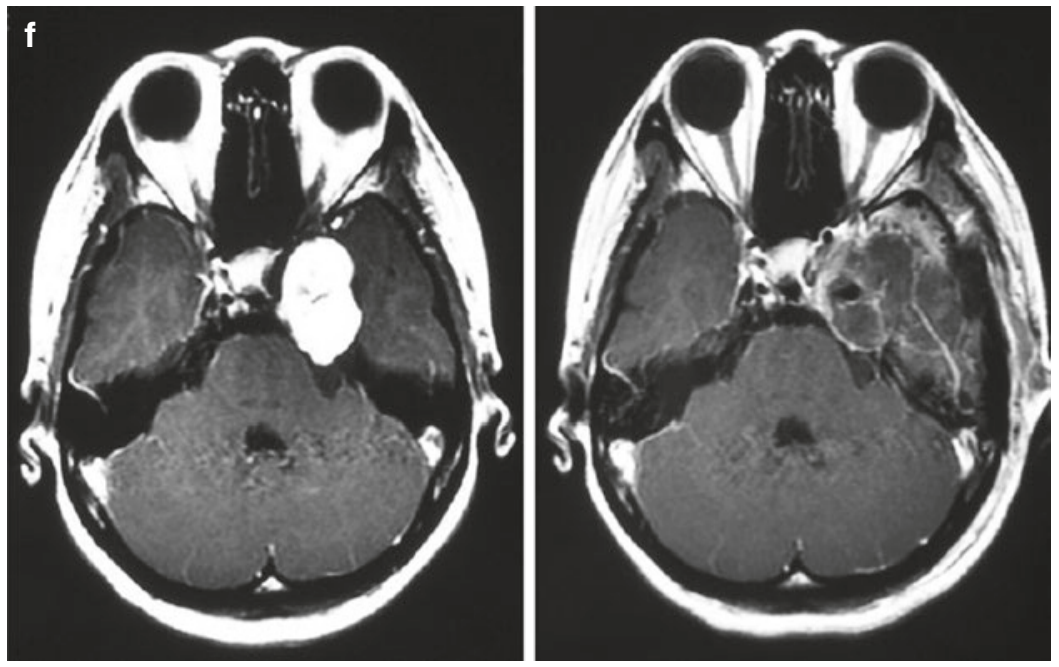

Trigeminal neurinomal17M

PostOp MRI

PreOp MRI
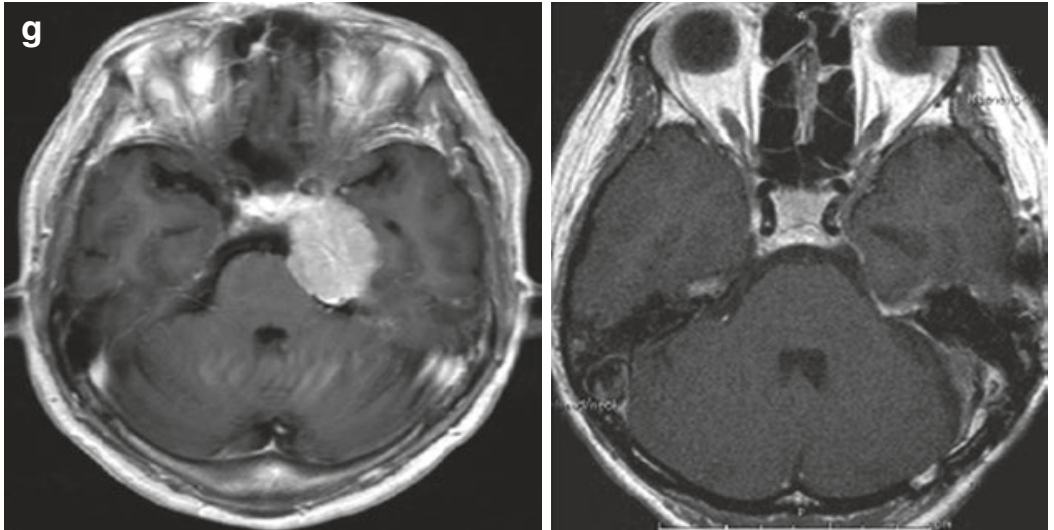

Meningioma

PreOp MRI

PostOp MRI

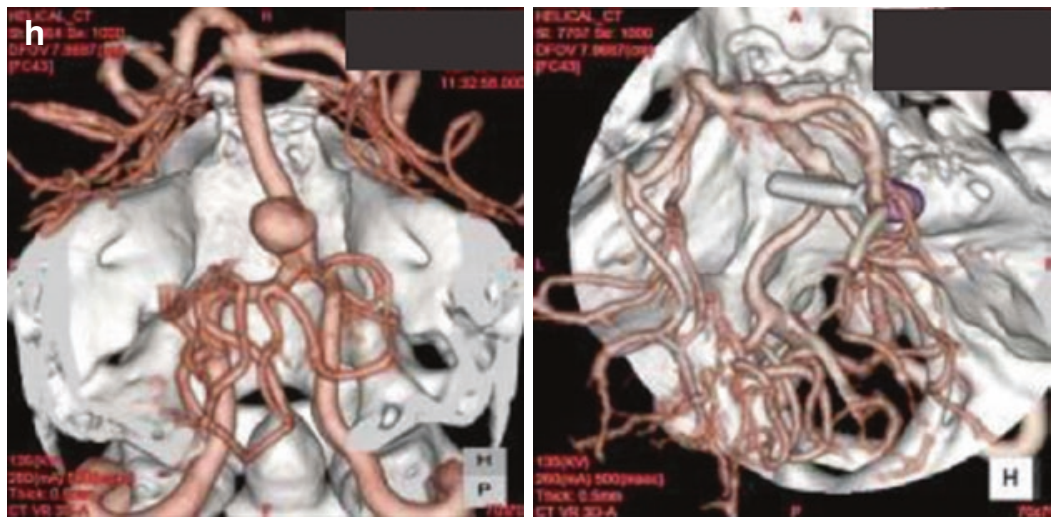

Basilar trunk aneurysm 54F

PreOp MRI

PostOp MRI

Fig. 9.7 (continued) 
Open Access This chapter is licensed under the terms of the Creative Commons Attribution 4.0 International License (http://creativecommons.org/licenses/by/4.0/), which permits use, sharing, adaptation, distribution and reproduction in any medium or format, as long as you give appropriate credit to the original author(s) and the source, provide a link to the Creative Commons license and indicate if changes were made.

The images or other third party material in this chapter are included in the chapter's Creative Commons license, unless indicated otherwise in a credit line to the material. If material is not included in the chapter's Creative Commons license and your intended use is not permitted by statutory regulation or exceeds the permitted use, you will need to obtain permission directly from the copyright holder.

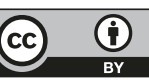

